\title{
Detection of a local Mycobacterium bovis reservoir using cattle surveillance data
}

\author{
Sara H. Downs ${ }^{1}$, Stuart Ashfield ${ }^{1}$, Mark Arnold ${ }^{1}$, Tony Roberts ${ }^{2}$, Alison Prosser ${ }^{1}$, Andy \\ Robertson $^{3}$, Susanne Frost ${ }^{2}$, Kate Harris ${ }^{1}$, Rachelle Avigad ${ }^{1}$, and Graham C. Smith ${ }^{4}$ \\ ${ }^{1}$ Animal and Plant Health Agency \\ ${ }^{2}$ Veterinary Advice Services Animal and Plant Health Agency Woodham Lane New Haw \\ Addlestone Surrey KT15 3NB UK \\ ${ }^{3}$ University of Exeter Environment and Sustainability Institute \\ ${ }^{4}$ National Wildlife Management Centre Animal and Plant Health Agency York UK
}

May 19, 2021

\begin{abstract}
The incidence of bovine tuberculosis (TB, caused by Mycobacterium bovis) in cattle has been associated with TB in badgers ( Meles meles) in parts of England. The aim was to identify badger associated M. bovis reservoirs in the Edge Area, between the High and Low Risk Areas for cattle TB. Data from badger TB surveys were sparse. Therefore, a definition for a local $M$. bovis reservoir potentially shared by cattle and badgers was developed using cattle TB surveillance data. The performance of the definition was estimated through Latent Class Analysis using badger TB survey data. Spatial units $\left(25 \mathrm{~km}^{2}\right)$ in the Edge Area were classified as having a reservoir if they had i) at least one OTF-W (Officially Tuberculosis Free - Withdrawn) incident in a cattle herd not attributed to cattle movement in the previous two years, ii) more OTF-W incidents than Officially Tuberculosis Free - Suspended (OTF-S) incidents in the previous two years and iii) at least one TB incident (OTF-S or OTF-W) in at least three of the previous seven years. Approximately twenty percent of the Edge Area was classified as having a local $M$. bovis reservoir using the cattle-based definition. Assuming 15\% TB prevalence in Edge Area badgers, sensitivity for the local M. bovis reservoir definition varied from $25.7 \%$ (95\% Credible Interval (CrI) 10.7 to $85.1 \%$ ) to $64.8 \%$ (95\% CrI 48.1 to 88.0 \%). Specificity was $91.9 \%$ (CrI 83.6 to $97.4 \%$ ). Over ninety percent of the local reservoir was in stable endemic TB areas identified through previous work and its spatial distribution was largely consistent with local veterinary knowledge. Uncertainty in the reservoir spatial distribution was explored through its recalculation in spatial units shifted in different directions. We recommend that the definition is re-evaluated as further data on badger infection with $M$. bovis becomes available.
\end{abstract}

\section{Hosted file}

Downs_Edge ResMain17May21v7.1.pdf available at https://authorea.com/users/414573/articles/ 522586-detection-of-a-local-mycobacterium-bovis-reservoir-using-cattle-surveillance-data 